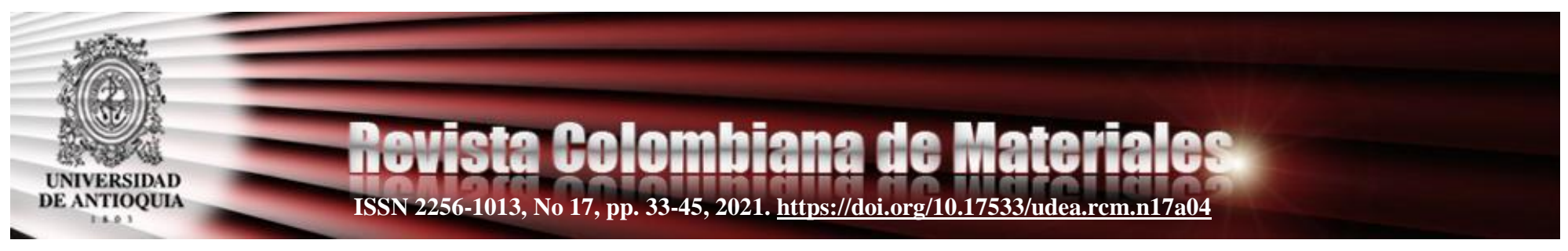

\title{
INFLUENCIA DE LA TEMPERATURA Y LA ATMOSFERA EN LA SINTERIZACIÓN DE LA HIDROXIAPATITA
}

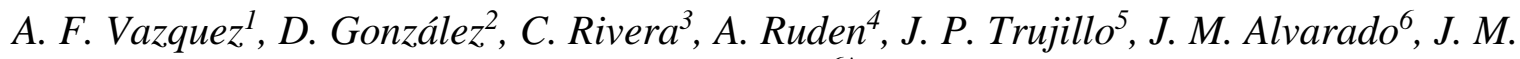 \\ González $^{6 *}$
}

1. M.Sc. Investigación y Desarrollo en New Stetic S.A, Medellín, Colombia.

2. Ph.D. Student in Materials Science and Engineering of Universidade Federal de São Carlos (UFSCar). Brazil.

3. Ingeniero, Laboratorio de Recubrimientos Duros y Aplicaciones Industriales. Universidad del Valle, Cali.

Colombia.

4. Ph.D. Docente, Grupo de Investigación: Ingeniería Biomédica y Ciencias Forenses. Facultad de Ciencias Básicas, Universidad Tecnológica de Pereira, Risaralda, Colombia.

5. M.Sc. Docente, Grupo de Investigación: Ingeniería Biomédica y Ciencias Forenses Facultad de Ciencias Básicas, Universidad Tecnológica de Pereira, Risaralda, Colombia.

6. Ph.D. CONACYT-Centro de Ingeniería y Desarrollo Industrial CIDESI-Consorcio de Manufactura Aditiva, CONMAD, Av. Pie de la Cuesta 702, Desarrollo San Pablo, Querétaro, México.

*juan.gonzalez@cidesi.edu.mx

\begin{tabular}{ll}
\hline PALABRAS CLAVE & RESUMEN \\
\hline Hidroxiapatita & En este trabajo se presenta el efecto de la temperatura y de la atmósfera de \\
Granulometría & sinterización de un polvo de Hidroxiapatita (HA) comercial en su composición \\
Sinterización & química, estructura cristalina, morfología y densidad. Se sinterizaron muestras \\
& compactadas de HA de aproximadamente $12 \mathrm{~mm}$ de diámetro y \\
& aproximadamente $0,3 \mathrm{~g}$ de peso en un rango de temperaturas entre 900 y 1200 \\
& ${ }^{\circ} \mathrm{C}$, con incrementos de $100{ }^{\circ} \mathrm{C}$, en atmósferas de aire y argón. Indiferente de \\
& la atmósfera de sinterización, la densidad relativa incrementó con la \\
& temperatura, mostrando mayor crecimiento de grano y reduciendo la cantidad \\
& de poros abiertos. Sin embargo, se observó un incremento en la pérdida de \\
& masa, con un efecto mayor para la atmósfera de argón. Como fue observado \\
& por espectroscopia infrarroja, el tratamiento en aire permite la rehidratación \\
del material en la etapa de enfriamiento, mientras que el análisis realizado por \\
medio de difracción de rayos X permitió demostrar que las muestras tratadas \\
en argón mostraron mayor grado de deshidroxilación y descomposición en $\beta-$ \\
TCP desde menores temperaturas que para las muestras sinterizadas en aire. \\
\hline
\end{tabular}




\section{INFLUENCE OF THE TEMPERATURE AND ATMOSPHERE IN THE SINTERING OF HYDROXYAPATITE}

\begin{tabular}{ll}
\hline KEYWORDS & ABSTRACT \\
\hline Hydroxyapatite & In This work the effect of the temperature and sintering atmosphere of commercial \\
Granulometry & Hydroxyapatite $(\mathrm{HA})$ powder on its chemical composition, crystal structure, \\
Sintering & morphology and density is presented. Compacted HA samples of approximately \\
& $12 \mathrm{~mm}$ in diameter and $\sim 0,3 \mathrm{~g}$ in weight were sintered at a temperature range \\
between 900 and $1200{ }^{\circ} \mathrm{C}$, with $100{ }^{\circ} \mathrm{C}$ increments, in atmospheres of air and \\
argon. Regardless of the sintering atmosphere, the relative density increased with \\
temperature, showing an increase in grain growth and reducing the open porosities. \\
Additionally, an increase in mass loss was observed, with a higher effect for the \\
argon atmosphere. As it is observed by infrared spectroscopy, the treatment in air \\
allows the rehydration of the material in the cooling stage, while the analysis \\
performed by X-ray diffraction demonstrated that the samples treated in argon \\
showed a higher degree of dehydroxylation and decomposition to $\beta$-TCP from \\
lower temperatures that for samples sintered in air.
\end{tabular}

\section{INTRODUCCIÓN}

La Hidroxiapatita (HA) es un mineral derivado de fosfatos cálcicos que ha recibido últimamente una gran atención en el campo de los biomateriales. Esto es debido a que este cerámico es químicamente similar al constituyente inorgánico principal del hueso natural [1] y por ende, presenta una gran afinidad con el organismo, además de ostentar inactividad química en condiciones fisiológicas y osteoactividad [2]. Por otra parte, la HA es un material bioactivo, lo cual permite la integración del mismo con estructuras óseas, facilitando el crecimiento del hueso e interactuando con el tejido vivo, debido a la presencia de compuestos libres de calcio y fósforo. Estas propiedades hacen a la Hidroxiapatita muy atractiva y ampliamente usada como material para implantes óseos [2]. Sin embargo, la descomposición térmica de la HA durante su sinterización afecta las propiedades fisicoquímicas del material [3]. $\mathrm{Se}$ ha reportado que los productos de descomposición poseen una tendencia mayor a la disolución y degradación que la HA en ambientes acuosos [4], lo cual implica una reducción en su estabilidad química, acelerando la degradación del material in vivo. Por esta razón el comportamiento térmico de la HA es de especial importancia para la utilización del cerámico en aplicaciones biomédicas. El comportamiento térmico de la HA ha sido descrito en dos etapas: deshidroxilación y descomposición $[5,6]$. La descomposición está precedida por la deshidroxilación, es decir, la pérdida de grupos $\mathrm{OH}$ - de la molécula del compuesto [7, 8], formando un compuesto meta-estable conocido como OxyHidroxiapatita (OHA) [7]. La descomposición de la HA, por consiguiente, resulta de una deshidroxilación que sobrepasa un punto crítico establecido por un rango de temperaturas fijadas normalmente entre 1200 y $1450{ }^{\circ} \mathrm{C}$ [5], donde la $\mathrm{HA}$ con una relación $\mathrm{Ca} / \mathrm{P}$ cercana a 1,67 es estable hasta los $1200{ }^{\circ} \mathrm{C}$. A temperaturas mayores ocurriría una deshidroxilación de carácter irreversible, resultando así en el colapso de la estructura de la HA al formar compuestos como fosfato tricálcico en diferentes estructuras cristalinas (TCP y TTCP) [9]. En el presente estudio se sinterizó un polvo comercial de Hidroxiapatita a cuatro diferentes temperaturas y dos atmósferas de 
trabajo. El objetivo es evaluar la estabilidad térmica de la HA en función de la temperatura y atmósfera de sinterización, basándose principalmente en análisis de Difracción de Rayos X, Espectroscopía Infrarroja y Microscopía Electrónica de Barrido.

\section{METODOLOGIA EXPERIMENTAL}

Se compactaron muestras de aproximadamente $0,3 \mathrm{~g}$ a partir de un polvo comercial de Hidroxiapatita (Strem Chemicals, INC.) en una matriz de compactación cilíndrica para trabajo en frío de $12 \mathrm{~mm}$ de diámetro interno, ejerciendo una presión de $100 \mathrm{MPa}$ por medio de una prensa mecánica. Posteriormente estas fueron sinterizadas en un horno de cámara cilíndrica (MTI Corporation, GSL-1500X) utilizando una tasa de calentamiento de $5{ }^{\circ} \mathrm{C} / \mathrm{min}$, un rango de temperaturas máximas de 900-1200 ${ }^{\circ} \mathrm{C}$ con aumentos de $100{ }^{\circ} \mathrm{C}$ y dos atmósferas de trabajo, aire y argón 99,99\% puro, con paso de flujo de 10 centímetros cúbicos por minuto (sccm). Las muestras fueron sostenidas a las máximas temperaturas durante 2 horas y enfriadas en el horno a una tasa de enfriamiento de $5{ }^{\circ} \mathrm{C} / \mathrm{min}$, para cada condición se realizaron 5 probetas. El polvo de HA comercial fue caracterizado granulométricamente por medio de Difracción Láser (DL), en un equipo de análisis de tamaño de partícula de referencia Malvern Instruments, modelo Mastersizer 2000, para lo cual se dispersó en una solución de Hexametafosfato de sodio al $10 \%$ en peso y posteriormente se expuso a ultrasonido. Se realizaron estudios de Difracción de Rayos X (DRX) tanto al polvo, como a las muestras sinterizadas para identificar las fases cristalinas que las constituyen, para lo cual se utilizó un difractómetro PANalytical modelo Xpert Powder con geometría Bragg Brentano, utilizando radiación de $\mathrm{CuKa}(\lambda=1,54056 \AA)$ $y$ un barrido de $10^{\circ}$ a $70^{\circ}$. Igualmente se efectuaron análisis de Espectroscopia de
Infrarrojo por Transformada de Fourier (FTIR) en un equipo de marca NICOLET 6700 FT-IR por el método KBr. Las pastillas de HA sinterizadas fueron trituradas $y$ posteriormente mezcladas con Bromuro de Potasio para ser compactadas y analizadas en el modo de transmitancia a lo largo del rango medio infrarrojo, en el intervalo de longitudes de onda entre 4000 y $400 \mathrm{~cm}^{-1}$. Por medio de Microscopía Electrónica de Barrido (MEB) se evaluó la morfología de los polvos y de las pastillas compactadas y sinterizadas a las diferentes temperaturas. Para ello se utilizó un Microscopio JEOL JSM-6490LV. Las micrografías permitieron constatar la densidad de las muestras, la cual se determinó siguiendo el procedimiento planteado en la norma ISO 18754:2020. Se tomaron las dimensiones de cada muestra con sus respectivos pesos y se obtuvo un valor de densidad por medio de la relación masa/volumen $\left(\mathrm{g} / \mathrm{cm}^{3}\right)$, este procedimiento se realizó por triplicado. La densidad relativa (\%) se obtuvo relacionando el valor obtenido con la densidad teórica de la Hidroxiapatita $\left(3,156 \mathrm{~g} / \mathrm{cm}^{3}\right)$.

\section{RESULTADOS Y DISCUSIÓN}

\subsection{Caracterización de la materia prima}

La Figura 1 muestra la distribución de los tamaños de partícula de los polvos de HA obtenida por granulometría de Difracción Láser. Se puede afirmar que la distribución de tamaños de partícula tiene una tendencia bimodal, donde el $90 \%$ de las partículas presentan un rango de tamaño de hasta 48,4 $\mu \mathrm{m}$ y el $10 \%$ restante se encuentra entre 48,4 $\mu \mathrm{m}$ y $114 \mu \mathrm{m}$. La polidispersión del polvo de HA permite una mejor distribución de las partículas durante el llenado del molde de compactación, puesto que las de menor tamaño pueden ocupar los espacios entre las partículas de mayor dimensión e incluso entre los aglomerados formados, aumentando así la 
densidad y la compresibilidad del polvo agregado en la matriz de compactación [5].

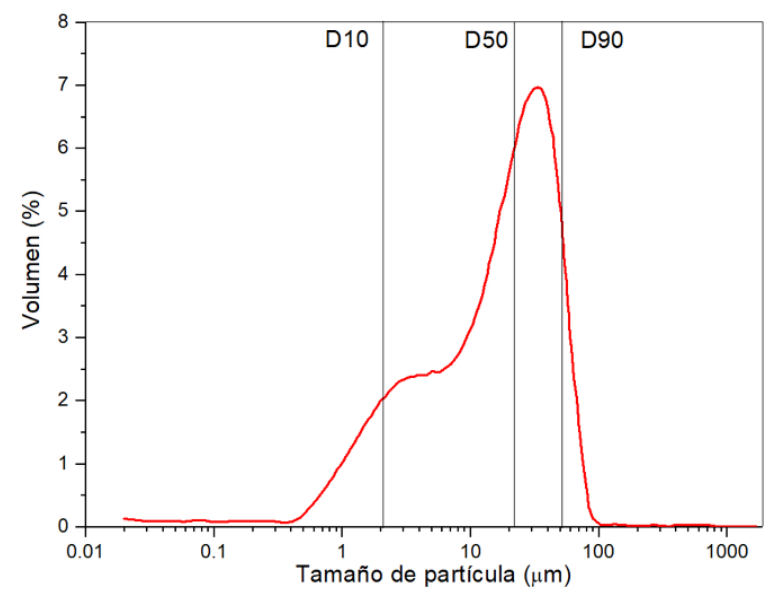

Figura 1. Distribución granulométrica de los polvos comerciales de $H A$.

El tamaño de partícula y el área específica superficial del polvo son parámetros relevantes a la hora de analizar la sinterización del material en verde (previo a la sinterización). H. Y. Juang \& M.H. Hon [10] demostraron que a medida que aumenta el tamaño medio de partícula se retrasa el inicio de la sinterización, por ende, es necesario un rango de temperatura más elevado para que ocurra una contracción volumétrica, puesto que la probabilidad de formación de cuellos disminuye con áreas superficiales menores. Una partícula más fina aporta un área superficial mucho mayor, lo cual aumenta la sinterabilidad del material, se reduce el tamaño de poro, pero al mismo tiempo reduce el factor de empaquetamiento del mismo [5]. El tamaño medio de partícula mayor utilizado por J.S Reed [11] fue de 4,19 $\mu \mathrm{m}$, el cual difiere ampliamente con el diámetro medio de la materia prima utilizada en este análisis $(22,14 \mu \mathrm{m})$. Se esperaría entonces la obtención de morfologías con mayor cantidad de poros y menor densidad a temperaturas de sinterización como $900{ }^{\circ} \mathrm{C}$ y $1000{ }^{\circ} \mathrm{C}$. La micrografía SEM del polvo (Figura 2) muestra la distribución de tamaño de partículas de forma aleatoria, mostrando una clara diferencia en sus dimensiones; las partículas presentan morfologías irregulares, angulares y se encuentran aglomeradas.

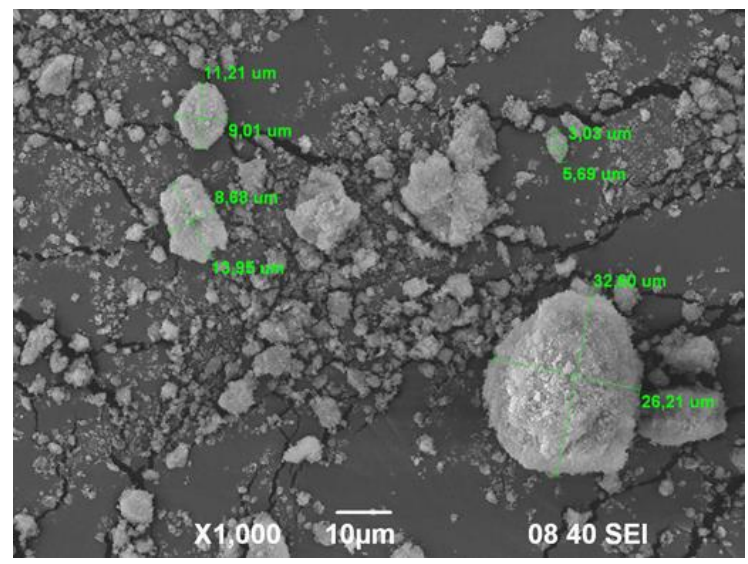

Figura 2. Micrografía SEM del polvo comercial de $H A$.

El análisis de DRX reveló una única fase de Hidroxiapatita en el polvo comercial, la cual está descrita con sus planos característicos sobre los picos de difracción y coincide con la carta PDF número 01-0759 526, ver Figura 3. No se encontraron otras fases típicas de los compuestos a base de fosfato de calcio, como fosfato tricálcico alfa $(\alpha-\mathrm{TCP})$, fosfato tricálcico beta $(\beta-\mathrm{TCP})$, o fosfato tetracálcico (TTCP), lo cual indica que no hay descomposición o mezcla de la HA con otros componentes cristalinos. El difractograma se encuentra de acuerdo con lo reportado por $S$. Ramesh et al. [12] para un polvo comercial de HA.

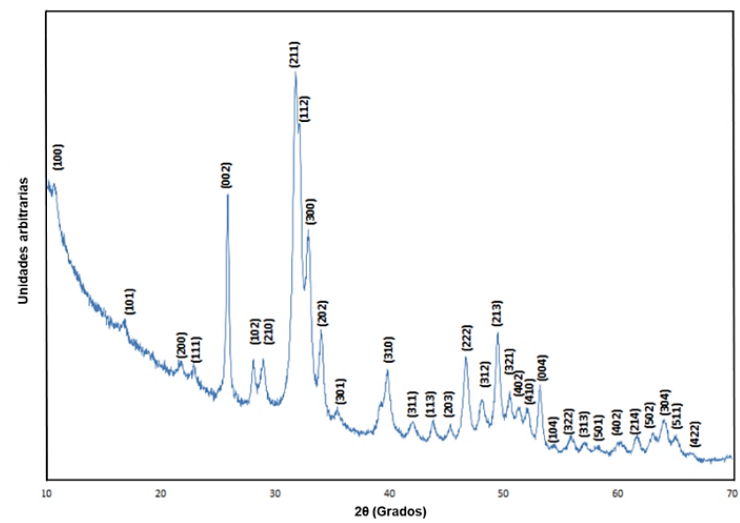

Figura 3. Patrón de Difracción de Rayos X de los polvos de HA comercial. 
El resultado del análisis FTIR correspondiente al polvo comercial, ver Figura 4, muestra un espectro característico de Hidroxiapatita con relación $\mathrm{Ca} / \mathrm{P}$ de aproximadamente 1,67 , el cual presenta bandas de vibraciones de los grupos funcionales $\mathrm{PO}_{4}{ }^{3-}$ y $\mathrm{OH}^{-}$, como lo presenta la Tabla 1.

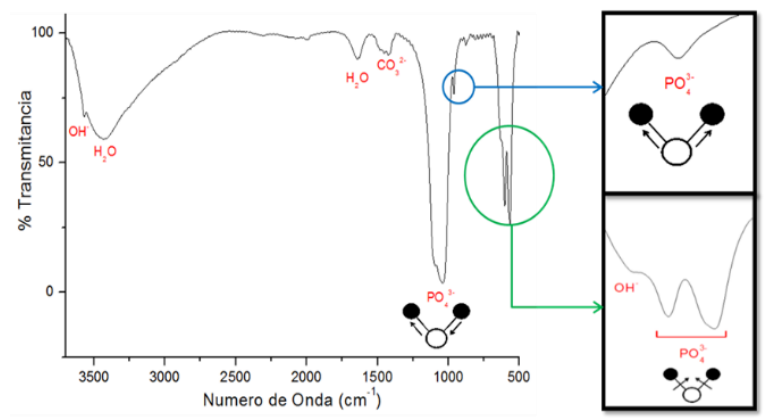

Figura 4. Espectro FTIR del polvo comercial de $H A$.

En el espectro también se observan las bandas correspondientes al $\mathrm{H}_{2} \mathrm{O}$ y $\mathrm{CO}_{3}{ }^{2-}$ localizados en números de onda $1500 \mathrm{~cm}^{-1}$ y $1600 \mathrm{~cm}^{-1}$ respectivamente, los cuales están relacionados con los procesos de absorción por exposición a la atmosfera.

Tabla 1. Bandas vibracionales encontradas en el espectro FTIR del polvo comercial de HA.

\begin{tabular}{|c|c|c|}
\hline $\begin{array}{c}\text { Grupos } \\
\text { funcionales }\end{array}$ & Bandas & $\begin{array}{c}\text { Número de } \\
\text { onda }\left(\mathbf{c m}^{-1}\right)\end{array}$ \\
\hline \multirow{3}{*}{$\mathrm{OH}^{-}$} & $v_{\text {Tensión }}(\mathrm{O}-\mathrm{H})$ & 3570 \\
\cline { 2 - 3 } & $v_{\text {Flexión }}(\mathrm{O}-\mathrm{H})$ & 630 \\
\hline \multirow{5}{*}{$\mathrm{PO}_{4}{ }^{3-}$} & $v_{1}(\mathrm{P}-\mathrm{O})$ & 962 \\
\cline { 2 - 3 } & $v_{2}(\mathrm{P}-\mathrm{O})$ & 472 \\
\cline { 2 - 3 } & $v_{3}(\mathrm{P}-\mathrm{O})$ & 1087 \\
\cline { 2 - 3 } & \multirow{2}{*}{$\mathrm{v}_{4}(\mathrm{P}-\mathrm{O})$} & 1046 \\
\cline { 3 - 3 } & & 600 \\
\hline
\end{tabular}

\subsection{Caracterización de compactados de Hidroxiapatita}

Las probetas sinterizadas en atmósfera de aire presentaron un cambio en su coloración, pasando de una tonalidad blanca a un azul perla, que se intensificó al aumentar la temperatura del proceso. La Figura 5 muestra el cambio en la coloración de las muestras, así como su cambio dimensional debido a la contracción volumétrica sufrida por el aumento en la temperatura.

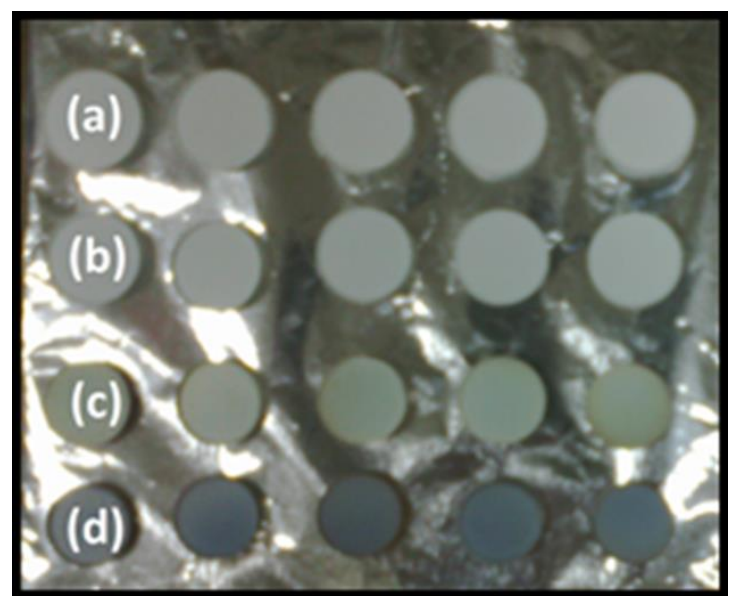

Figura 5. Muestras de HA sinterizadas en atmósfera de aire a diferentes temperaturas. (a) $900{ }^{\circ} \mathrm{C}$. (b) $1000{ }^{\circ} \mathrm{C}$. (c) $1100{ }^{\circ} \mathrm{C}$. (d) $1200{ }^{\circ} \mathrm{C}$.

El cambio en la tonalidad podría deberse a trazas o impurezas en el compuesto; L. Yubao et al. [13] atribuye este fenómeno a la oxidación del ion $\mathrm{Mn}^{2+}$, contenido por la $\mathrm{HA}$ en forma de impureza, produciendo $\mathrm{Mn}^{5+} \mathrm{y} / \mathrm{o}$ $\mathrm{MnO}_{4}{ }^{3-}$. La coloración de la Hidroxiapatita con trazas de manganeso está relacionada con la estructura cristalina de la HA sinterizada en presencia de atmósferas oxidantes, como el aire. Las temperaturas altas también son un factor decisivo; elevadas temperaturas de proceso incrementan tanto el poder oxidante de la atmósfera, como la energía de los iones oxidados de Mn. La disminución en el tamaño de las muestras sinterizadas es evidente como se muestra en la Tabla 2. La contracción volumétrica es propia de un material poroso altamente higroscópico, tal y como lo es la HA. No se encontraron grandes diferencias entre las probetas sinterizadas en aire y argón, salvo una pequeña desigualdad en sus dimensiones, esto es, las muestras tratadas térmicamente en atmósfera de gas inerte presentan diámetros y espesores levemente mayores a las sinterizadas en aire. De igual 
forma, no hay una diferencia significativa en las masas de las probetas sinterizadas, salvo si se comparan con la muestra en verde. Sin embargo, sí existe una tendencia a la pérdida de masa conforme aumenta la temperatura de sinterización. Esto podría justificarse con la disipación de vapor de agua absorbida por el material y/o la pérdida de iones hidroxilo $\left(\mathrm{OH}^{-}\right)$de la molécula. Podría afirmarse entonces que las muestras sinterizadas a 1200 ${ }^{\circ} \mathrm{C}$ en ambas atmósferas son las que sufren en mayor intensidad por la pérdida de agua y grupos $\mathrm{O}-\mathrm{H}$, con un mayor efecto en la atmósfera de argón, lo cual favorece la descomposición de la Hidroxiapatita en otros fosfatos de calcio.

Tabla 2. Dimensiones y masa de las muestras sinterizadas.

\begin{tabular}{|c|c|c|c|c|c|}
\hline Atmósfera & $\begin{array}{c}\text { Temperatura } \\
\left({ }^{\circ} \mathbf{C}\right)\end{array}$ & $\begin{array}{c}\text { Diámetro } \\
(\mathbf{m m})\end{array}$ & $\begin{array}{c}\text { Espesor } \\
(\mathbf{m m})\end{array}$ & Masa (g) & $\begin{array}{c}\text { Pérdida de } \\
\text { masa (\%) }\end{array}$ \\
\hline \multirow{2}{*}{ N/A } & Verde & $12,00 \pm 0,36$ & $1,42 \pm 0,13$ & $0,299 \pm 0,03$ & 0.00 \\
\hline \multirow{4}{*}{ Aire } & 900 & $11,60 \pm 0,34$ & $1,31 \pm 0,12$ & $0,275 \pm 0,02$ & 8,02 \\
\cline { 2 - 6 } & 1000 & $10,90 \pm 0,32$ & $1,31 \pm 0,10$ & $0,275 \pm 0,03$ & 8,02 \\
\cline { 2 - 6 } & 1100 & $10,20 \pm 0,31$ & $1,21 \pm 0,11$ & $0,270 \pm 0,02$ & 9,69 \\
\cline { 2 - 6 } & 1200 & $9,90 \pm 0,29$ & $1,21 \pm 0,09$ & $0,268 \pm 0,02$ & 10,36 \\
\hline \multirow{3}{*}{ Argón } & 900 & $11,80 \pm 0,35$ & $1,32 \pm 0,10$ & $0,275 \pm 0,02$ & 8,02 \\
\cline { 2 - 6 } & 1000 & $10,90 \pm 0,32$ & $1,32 \pm 0,12$ & $0,270 \pm 0,03$ & 9,69 \\
\cline { 2 - 6 } & 1100 & $10,3 \pm 0,30$ & $1,21 \pm 0,11$ & $0,268 \pm 0,02$ & 10,36 \\
\cline { 2 - 6 } & 1200 & $9,90 \pm 0,29$ & $1,21 \pm 0,09$ & $0,260 \pm 0,02$ & 13,04 \\
\hline
\end{tabular}

La Figura 6 presenta los espectros de FTIR donde se observan las vibraciones moleculares típicas de la HA para las muestras sinterizadas en ambas atmósferas. Al examinar los espectros del material tratado en aire, se encontraron bandas asignadas al enlace O-H (3571 y $630 \mathrm{~cm}^{-1}$ ) junto con las bandas típicas de la humedad adsorbida, lo que indica existencia de rehidratación durante el tiempo de enfriamiento en el horno. Sin embargo, hay una clara disminución en la intensidad con respecto al aumento de la temperatura de proceso, lo que indica que mayores temperaturas de sinterización produce menores grados de rehidratación del material. A $1200{ }^{\circ} \mathrm{C}$ desaparecen casi totalmente las bandas correspondientes al agua (3250-3700 y $\left.1600 \mathrm{~cm}^{-1}\right)$, lo que supondría que la Hidroxiapatita ha empezado a sufrir deshidroxilación irreversible. Por otra parte, la HA sinterizada a 1000, 1100 y 1200
${ }^{\circ} \mathrm{C}$ en atmósfera de argón presentó una pérdida mayor de iones hidroxilo en su estructura molecular, representada por la desaparición de las bandas correspondientes al agua adsorbida, lo que podría explicar la mayor diferencia en la masa de las muestras procesada en esta atmósfera. Con respecto al pico situado a $630 \mathrm{~cm}^{-1}$ (correspondiente a la banda de flexión del radical $\mathrm{OH}^{-}$), es clara su existencia en la muestra sinterizada a $900{ }^{\circ} \mathrm{C}$ y la menor intensidad en la tratada a $1000{ }^{\circ} \mathrm{C}$. Sin embargo, para las muestras sinterizadas a $1100{ }^{\circ} \mathrm{C}$ y $1200{ }^{\circ} \mathrm{C}$, la banda desaparece completamente. Igualmente, la muestra sinterizada a $1200^{\circ} \mathrm{C}$ en atmósfera de argón presenta un ensanchamiento de la banda vibracional entre 950 y $1200 \mathrm{~cm}^{-1}$, lo cual supone una descomposición del material, como lo indican estudios de A. Rapacz-Kmita et al. [5] y Y. Liu \& Z. Shen [12]. Dicha descomposición está relacionada con la 
liberación de grupos $\mathrm{OH}^{-}$y la formación de OxyHidroxiapatita (OHA). Para que dicha pérdida sea irreversible, dos de las moléculas monovalentes de $\mathrm{OH}^{-}$son reemplazadas por un oxígeno bivalente y una vacancia.
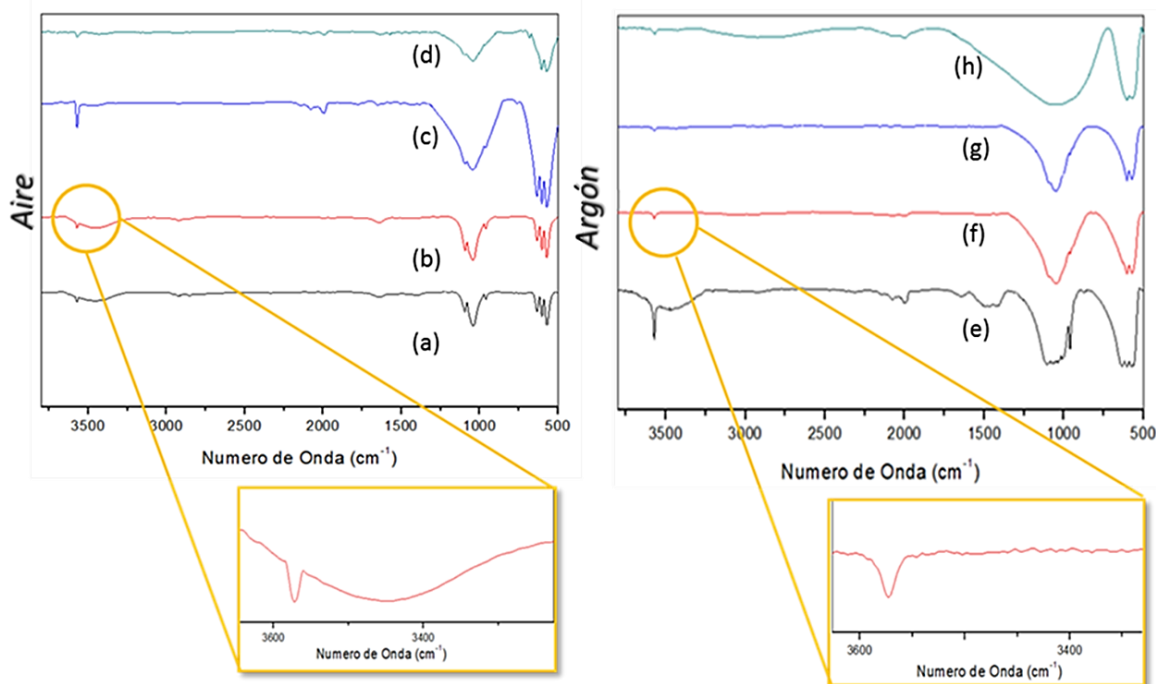

Figura 6. Espectros FTIR de las muestras sinterizadas en atmósfera de Aire y Argón. (a) $900{ }^{\circ} \mathrm{C}$ aire, (b) $1000{ }^{\circ} \mathrm{C}$ aire, (c) $1100{ }^{\circ} \mathrm{C}$ aire, (d) $1200{ }^{\circ} \mathrm{C}$ aire, (e) $900{ }^{\circ} \mathrm{C}$ argón, (f) $1000{ }^{\circ} \mathrm{C}$ argón, $(\mathrm{g}) 1100{ }^{\circ} \mathrm{C}$ argón,

(h) $1200{ }^{\circ} \mathrm{C}$ argón.

La Figura 7 presenta los picos de difracción para el material sinterizado en aire. Los resultados muestran la existencia de HA como constituyente primario y no se encontraron indicios de fases cristalinas como $\mathrm{CaO}, \alpha$-TCP o TTCP. Sin embargo, en el difractograma de la HA sinterizada a $1200{ }^{\circ} \mathrm{C}$ se identificó un pico de baja intensidad correspondiente al compuesto $\beta$-TCP $\left[\mathrm{Ca}_{3}\left(\mathrm{PO}_{4}\right)_{2}\right]$, el cual coincide con la carta PDF número 00-009-0346. Tal y como mostró el espectro FTIR, hubo una pérdida crítica de grupos $\mathrm{OH}^{-}$de la molécula, lo cual podría haber acelerado la descomposición de la Hidroxiapatita sinterizada a estas condiciones.

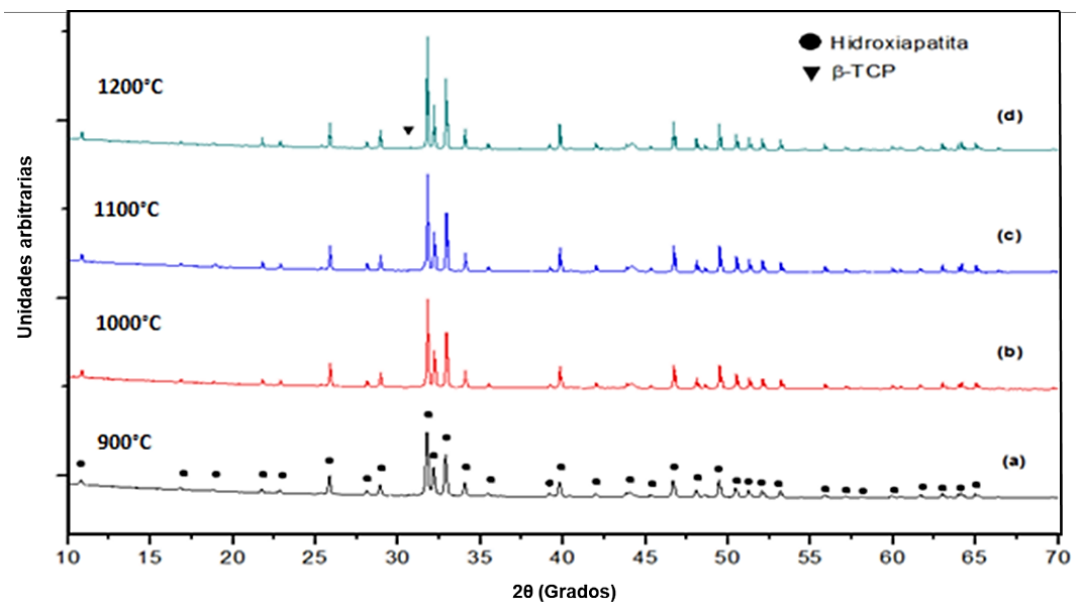

Figura 7. Picos de Difracción para la HA sinterizada en la atmósfera de aire. (a) $900{ }^{\circ} \mathrm{C}$. (b) $1000^{\circ} \mathrm{C}$. (c) $1100^{\circ} \mathrm{C}$. (d) $1200^{\circ} \mathrm{C}$. 
Tal como en el material tratado sin control de atmósfera, las muestras sinterizadas en argón presentaron un patrón de difracción correspondiente a la HA en todas las temperaturas (Figura 8). De forma similar a lo observado en atmósfera oxidante, para una temperatura de $1200{ }^{\circ} \mathrm{C}$ hay un pico de baja intensidad $\left(2 \theta=31,01^{\circ}\right)$, correspondiente al plano (2 0 10) del fosfato de calcio beta, el cual puede también observarse en la muestra sinterizada a $1100{ }^{\circ} \mathrm{C}$. Esto no es deseable para algunas aplicaciones biomédicas como las dentales y las de andamiaje celular, dado que compuestos como el fosfato tricálcico y el tetracálcico, presentan una mayor tendencia a disolverse, lo que disminuiría su estabilidad química [9, 14]. Según lo observado, la deshidroxilación es irreversible debido a la aparición de un pico de $\beta$-TCP en la HA en atmósfera de argón a menores temperaturas, contrario a lo que ocurre en atmósfera de aire, donde el mismo proceso se presenta en la temperatura de transición según el diagrama de fases [15].

En este caso, la atmósfera de argón favorece la descomposición de la hidroxipatita, lo que concuerda con lo encontrado por J.A. Lenis et al. [16] y B. Nasiri-Tabrizi et al. [17], donde se demuestra que el control de la atmósfera aumenta las probabilidades de deshidroxilación, debido a que no existe humedad del aire que ayude a la rehidratación de la HA durante el proceso de sinterización. Los autores depositaron recubrimientos $\mathrm{y}$ sinterizaron muestras respectivamente en atmósferas de aire y argón, confirmando así que durante la sinterización en argón la deshidroxilación se presenta a menor temperatura que en aire. Por otra parte, $S$. Ramesh et al. [12], afirman que el grado de deshidroxilación y descomposición de la HA depende, entre otros factores, de la presión parcial del agua en la atmósfera de sinterización. Al existir un alto contenido de humedad en el ambiente, se reduce la tendencia a la descomposición, evitando la deshidratación severa del material.

Las muestras sinterizadas en argón a 900 y $1000{ }^{\circ} \mathrm{C}$ no evidenciaron deshidroxilación quizás porque dichas temperaturas son cercanas a la del inicio de la pérdida de iones hidroxilo en la HA [7] y por lo tanto, la energía aportada solo produjo la liberación de vapor de agua en la superficie.

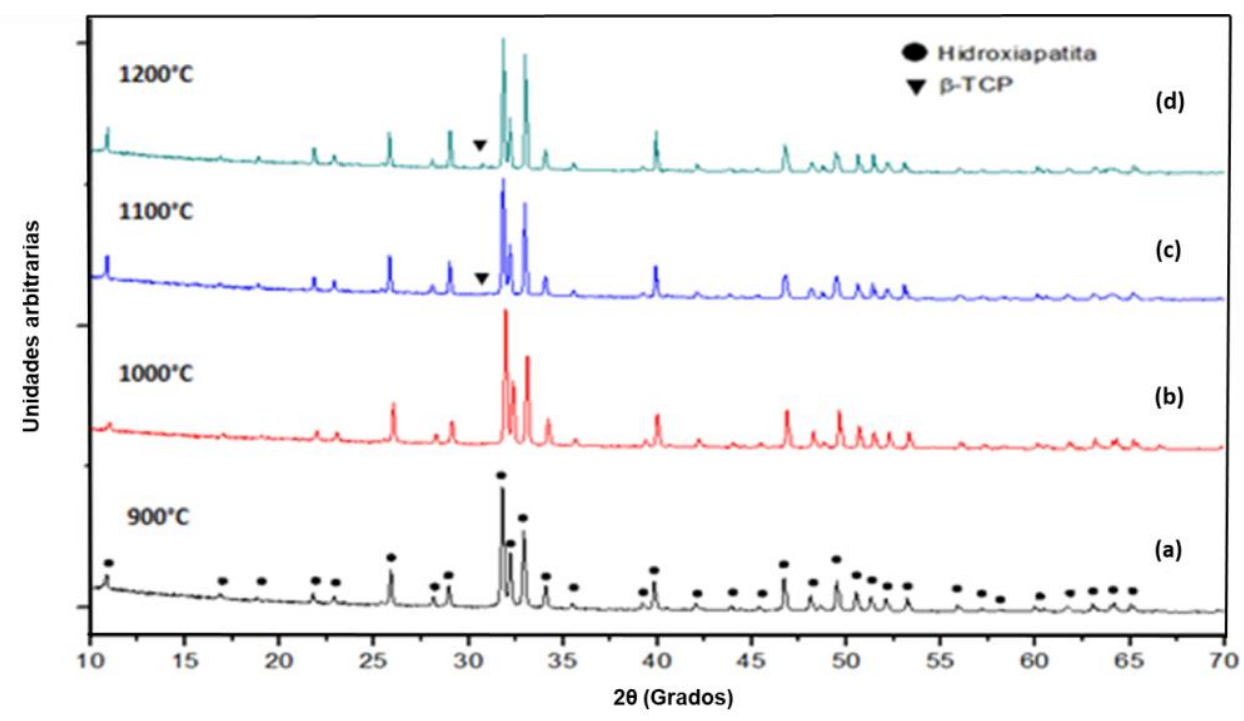

Figura 8. Picos de Difracción para la HA sinterizada en atmósfera de argón. (a) $900^{\circ} \mathrm{C}$. (b) $1000^{\circ} \mathrm{C}$. (c) $1100^{\circ} \mathrm{C}$. (d) $1200^{\circ} \mathrm{C}$. 
Por medio de Microscopía Electrónica de Barrido (Figura 9), fue posible observar el incremento de la homogeneización de las muestras sinterizadas en aire conforme se aumenta la temperatura de sinterización. A $900{ }^{\circ} \mathrm{C}$ se observa una densificación parcial, puesto que esta temperatura no aporta suficiente energía para lograr una difusión significativa, es decir, sólo son evidentes las formaciones de cuellos entre las partículas, precedidas por un alisamiento superficial, $\mathrm{La}$ muestra sinterizada en estas condiciones presenta una porosidad superficial elevada, típica de una estructura en su etapa inicial de sinterización [18]. Para la muestra sinterizada a $1000{ }^{\circ} \mathrm{C}$ se puede observar un mayor grado de densificación, lo que se caracteriza por la reducción parcial de la porosidad y un crecimiento lento de grano [18]. La muestra tratada a $1100{ }^{\circ} \mathrm{C}$ presenta una estructura granular más definida, conformada por granos de mayor tamaño, poros abiertos y cerrados, debido al encogimiento de los poros abiertos ubicados en la intersección de los bordes de grano [18]. Por último, la muestra sinterizada a $1200{ }^{\circ} \mathrm{C}$ exhibe un mayor crecimiento del tamaño de grano, aumentando la homogeneización superficial por la disminución de poros abiertos.
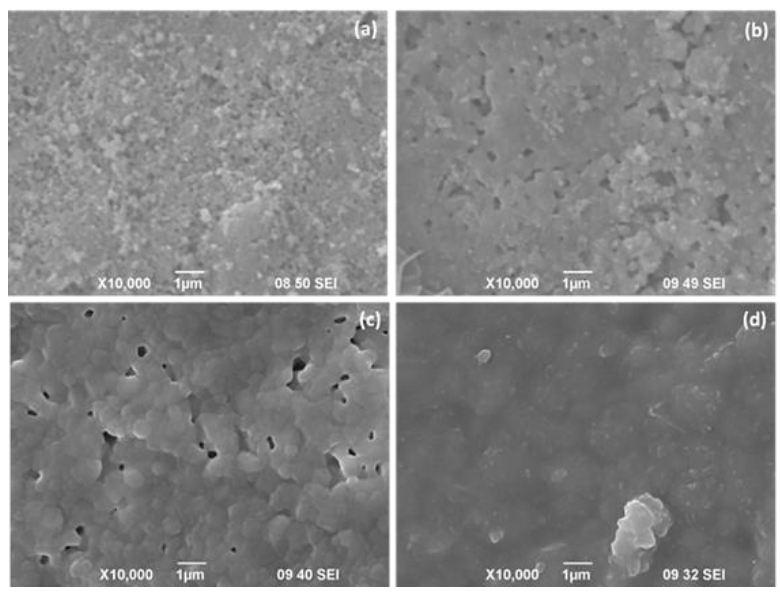

Figura 9. Micrografías SEM a 10000X para las muestras sinterizadas en aire. (a) $900{ }^{\circ} \mathrm{C}$, (b) $1000{ }^{\circ} \mathrm{C}$, (c) $1100{ }^{\circ} \mathrm{C}, y(d) 1200{ }^{\circ} \mathrm{C}$.
Para el caso de la HA sinterizada en argón (Figura 10), el efecto de la temperatura en la morfología tiene un comportamiento similar a la HA sinterizada en aire; en éstas también se logra apreciar que el aumento de la temperatura promueve el proceso de densificación superficial. A pesar de que la tendencia a la densificación con el aumento de la temperatura es similar para ambos tratamientos, la intensidad o el grado de la homogeneización no es el mismo; se puede apreciar una leve diferencia en las porosidades entre los materiales sinterizados a las mismas temperaturas. Las muestras tratadas en aire exhiben un grado de porosidad menor, en contraste con las sinterizadas en argón. Los granos observados en las muestras sinterizadas en aire presentan menores tamaños respecto a las sinterizadas en argón, lo que produciría mayor superficie de contacto $\mathrm{y}$, por tanto, mayor interacción, crecimiento y coalescencia [18].
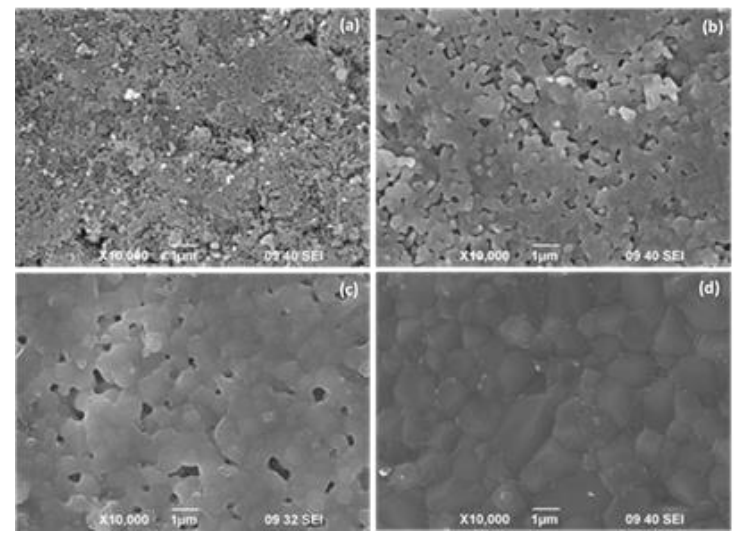

Figura 10. Micrografías SEM a 10000x para las muestras sinterizadas en argón. (a) $900^{\circ} \mathrm{C}$, (b) $1000{ }^{\circ} \mathrm{C}$, (c) $1100{ }^{\circ} \mathrm{C}, y(d) 1200{ }^{\circ} \mathrm{C}$.

Caso contrario ocurre con las muestras sinterizadas en argón, que exhiben granos de mayor tamaño, reduciendo el área de contacto y la interacción entre granos, produciendo una superficie menos densa. La diferencia entre las morfologías de las muestras sinterizadas a iguales temperaturas, pero distintas atmósferas podrían explicarse analizando el grado de deshidroxilación. 
Como se indicó anteriormente, las muestras sinterizadas en argón presentaron una mayor deshidratación que las sinterizadas de aire.

Los valores obtenidos para la densidad y densidad relativa de las muestras antes y después de los tratamientos de sinterización son mostrados en la Tabla 3. Los valores de densidad demuestran una tendencia a aumentar conforme se eleva la temperatura de sinterización. La densidad relativa de las muestras en verde, así como la de las muestras sinterizadas a $900{ }^{\circ} \mathrm{C}$ en aire y argón es casi el $60 \%$ del valor teórico de la Hidroxiapatita. El bajo porcentaje de contracción y la pérdida mínima de masa para las muestras tratadas a esta temperatura hacen que las densidades sean similares a la de la probeta en verde y comprueban que en el tratamiento térmico de sinterización realizado a $900{ }^{\circ} \mathrm{C}$ las muestras no experimentan un elevado grado de densificación y tampoco una pérdida importante en el porcentaje de agua adsorbida y de grupos $\mathrm{OH}^{-}$.
La relación entre la densidad y la temperatura de sinterización se muestra en la Figura 11. Se evidencia una leve diferencia entre las densidades de la HA sinterizada en atmósfera de aire y de argón. Como se discutió anteriormente, las muestras sinterizadas en argón presentaron un nivel de deshidroxilación mayor a las tratadas en aire, lo que claramente, según T. P. Hoepfner \& E. $D$ Case [19], pudo afectar la densificación de la HA sinterizada bajo atmósfera controlada. La densidad aproximada de las muestras presenta un comportamiento típico de una función sigmoide (Figura 11), es decir, un incremento periódico suave en una primera etapa, seguido de un crecimiento cuasi-lineal y rescindiendo en una atenuación y estabilización de la función. Este comportamiento fue reportado por $G$. Muralithran \& S. Ramesh. [20] y S. Ramesh et ál. [12], donde la densidad de la HA también exhibe este comportamiento.

Tabla 3. Densidad y densidad relativa de las muestras de HA compactada y sinterizadas.

\begin{tabular}{|c|c|c|c|c|c|}
\hline Atmósfera & Temp $\left({ }^{\circ} \mathbf{C}\right)$ & Volumen $\left(\mathbf{c m}^{\mathbf{3}}\right)$ & Masa $(\mathbf{g})$ & $\begin{array}{c}\text { Densidad } \\
\left(\mathbf{g} / \mathbf{c m}^{\mathbf{3}}\right)\end{array}$ & $\begin{array}{c}\text { Densidad } \\
\text { Relativa (\%) }\end{array}$ \\
\hline N/A & Verde & $0,161 \pm 0,014$ & $0,299 \pm 0,023$ & 1,862 & 58,99 \\
\hline \multirow{4}{*}{ Aire } & 900 & $0,138 \pm 0,012$ & $0,275 \pm 0,022$ & 1,986 & 62,94 \\
\cline { 2 - 6 } & 1000 & $0,122 \pm 0,010$ & $0,275 \pm 0,021$ & 2,250 & 71,28 \\
\cline { 2 - 6 } & 1100 & $0,099 \pm 0,008$ & $0,270 \pm 0,022$ & 2,731 & 86,53 \\
\cline { 2 - 6 } & 1200 & $0,093 \pm 0,008$ & $0,268 \pm 0,008$ & 2,877 & 91,17 \\
\hline \multirow{3}{*}{ Argón } & 900 & $0,144 \pm 0,012$ & $0,275 \pm 0,012$ & 1,905 & 60,36 \\
\cline { 2 - 6 } & 1000 & $0,123 \pm 0,011$ & $0,270 \pm 0,021$ & 2,192 & 69,46 \\
\cline { 2 - 6 } & 1100 & $0,101 \pm 0,009$ & $0,268 \pm 0,022$ & 2,658 & 84,23 \\
\cline { 2 - 6 } & 1200 & $0,093 \pm 0,008$ & $0,260 \pm 0,020$ & 2,791 & 88,45 \\
\hline
\end{tabular}




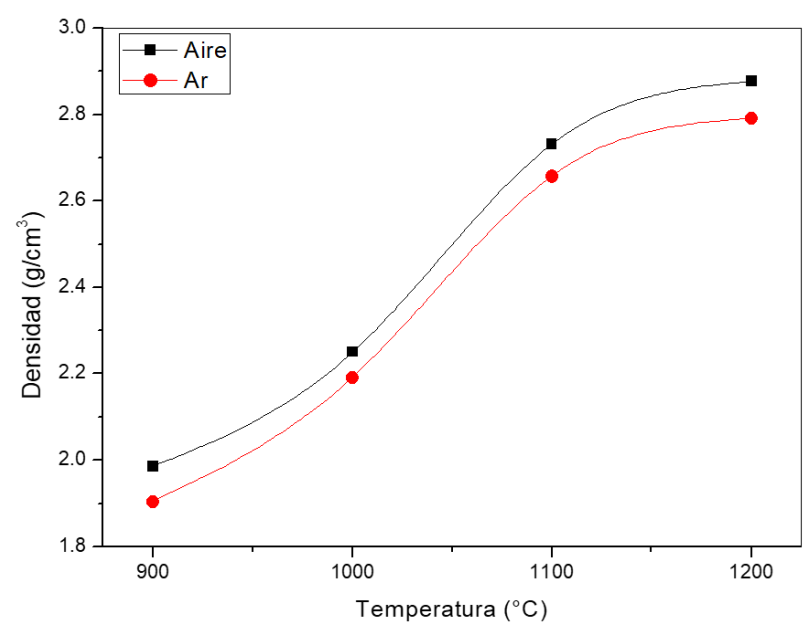

Figura 11. Densidad de las muestras sinterizadas en función de su temperatura de sinterización.

\section{CONCLUSIONES}

Los resultados de los análisis realizados mediante DRX y FTIR permitieron comprobar que el polvo utilizado para este estudio está constituido por Hidroxiapatita $\left[\mathrm{Ca}_{10}\left(\mathrm{PO}_{4}\right)_{6}(\mathrm{OH})_{2}\right]$, como única fase cristalina. Es decir, no se encontraron evidencias de fases cristalinas secundarias ni de descomposición de la Hidroxiapatita. La atmósfera de sinterización afecta sensiblemente la estabilidad estructural de la HA para el rango de temperaturas estudiadas. Las muestras tratadas en argón y a temperaturas mayores a $900{ }^{\circ} \mathrm{C}$ presentaron deshidroxilación (pérdida de grupos $\mathrm{OH}^{-}$) en comparación con las probetas sinterizadas en aire. Esto es debido a la carencia de humedad propia del ambiente, lo cual impidió la rehidratación del compuesto durante el enfriamiento. Los resultados del análisis de DRX revelaron indicios de descomposición a $1100{ }^{\circ} \mathrm{C}$ en atmósfera de argón, al igual que en las muestras sinterizadas a $1200{ }^{\circ} \mathrm{C}$ (en ambas atmósferas), las cuales presentaron picos correspondientes a $\beta$-TCP, fase característica de la degradación de la HA en altas temperaturas, la cual posee un mayor grado de biodegradabilidad, pues es más reactiva en medios acuosos. El análisis realizado utilizando MEB y las medidas de densidad, corroboró el incremento del grado de densificación de las muestras con respecto a la temperatura. La HA sinterizada a $1200{ }^{\circ} \mathrm{C}$ en ambas atmósferas mostró una microestructura más compacta a partir de granos de tamaños mayores. Las probetas sinterizadas en atmósfera de aire presentaron un menor grado de porosidad, y por ende mayores densidades.

\section{AGRADECIMIENTOS}

Los autores agradecen al Grupo de Investigación Recubrimientos Duros y Aplicaciones Industriales, Universidad del Valle, Colombia, en especial a su director Dr Federico Sequeda por su colaboración en el presente trabajo. A los profesores Pedro José Arango, de la Universidad Nacional de Colombia sede Manizales y Gilberto Bolaños Pantoja de UNICAUCA. Juan Manuel González Carmona agradece al programa de Cátedras CONACyT por el financiamiento otorgado durante la realización de esta investigación. Agradecimiento a los organizadores del Primer Simposio Nacional de Prótesis Ortopédicas el Estatus Actual en México.

\section{REFERENCIAS}

[1] R. Singh Soni \& V. Pratap Singh. Fabrication and experimental analysis of hydroxyapatite based composite materials for medical implants, Materials Today: Proceedings 2020.

[2] P. Madhavasarma. P. Veeraragavan, S. Kumaravel, M. Sridevi. Studies on physiochemical modifications on biologically important hydroxyapatite materials and their characterization for medical applications, 2020. Biophys. Chem. 267, 106474.

[3] D. He, X. Zhang, P. Liu, X. Liu, X. Chen, F. Ma, W. Li, K. Zhang, H. Zhou. Effect of 
hydrothermal treatment temperature on the hydroxyapatite coatings deposited by electrochemical method, 2021. Surf. Coat. Technol. 406, 126656.

[4] S. Bhattacharjee, S. K. Swain, D. K. Sengupta, B. P. Singh. Effect of heat treatment of hydroxyapatite on its dispersibility in aqueous medium, 2006. Colloids Surf. A Physicochem. Eng. Asp. 277, 164-170.

[5] A. Rapacz-Kmita, C. Paluszkiewicz, A. Ślósarczyk, Z. Paszkiewicz. FTIR and XRD investigations on the thermal stability of hydroxyapatite during hot pressing and pressureless sintering processes, 2005. J. Mol. Struct. 744-747, 653-656.

[6] T. Wang, A. Dorner-Reisel. Thermoanalytical investigations of the decomposition of oxyhydroxyapatite, 2004. Mater. Lett. 58, 3025-3028.

[7] Y. Liu, Z. Shen. Dehydroxylation of hydroxyapatite in dense bulk ceramics sintered by spark plasma sintering, 2012. J. Eur. Ceram. Soc. 32.

[8] S. Laasri, M. Taha, A. Laghzizil, E. K. Hlil. J.Chevalier The affect of densification and dehydroxylation on the mechanical properties of stoichiometric hydroxyapatite bioceramics, 2010. . Mater. Res. Bull. 45, 1433-1437.

[9] C. Rey, C. Combes, Drouet, C, C. Somrani. Tricalcium phosphate-based ceramics, 2008. in: Bioceramics and Their Clinical Applications. Woodhead Publishing, pp. 326-366.

[10] H.Y. Juang, M.H. Hon. Effect of calcination on sintering of hydroxyapatite. Biomaterials 17, 1996, 2059-2064.
[11] J.S. Reed, 1989. Introduction to the Principles of Ceramic Processing.

[12] S. Ramesh, C. Y. Tan, R. Tolouei, M. Amiriyan, J. Purbolaksono, I. Sopyan, W. D. Teng. Sintering behavior of hydroxyapatite prepared from different routes, 2012. Mater. Des. 34, 148-154.

[13] L. Yubao, C. P. Klein, X. Zhang, K. de Groot, 1993. Relationship between the colour change of hydroxyapatite and the trace element manganese. Biomaterials 14, 969972.

[14] C. J. Liao, F. H. Lin, K. S. Chen, J. S. Sun, 1999. Thermal decomposition and reconstitution of hydroxyapatite in air atmosphere. Biomaterials 20, 1807-1813.

[15] P.W. Brown, B. Constantz, 2017. Hydroxyapatite and Related Materials. CRC Press. Calcium Phosphate Bioceramics and Cements, 2019. in: Principles of Regenerative Medicine. Academic Press, pp. 591-611.

[16] J. A. Lenis, F. M. Hurtado, M. A. Gómez, F. J. Bolívar. Effect of thermal treatment on structure, phase and mechanical properties of hydroxyapatite thin films grown by RF magnetron sputtering, 2019. Thin Solid Films 669, 571-578.

[17] B. Nasiri-Tabrizi, A. Fahami, R. Ebrahimi-Kahrizsangi Effect of milling parameters on the formation of nanocrystalline hydroxyapatite using different raw materials, 2013. Ceram. Int. 39, 5751-5763.

[18] H-Z. Shen, N. Guo, Y-H. Liang, P. Shen. Synthesis and densification of hydroxyapatite by mechanochemically-activated reactive cold sintering, 2021. Scr. Mater. 194, 113717.

[19] T. P. Hoepfner, E. D Case, The influence of the microstructure on the hardness of 
sintered hydroxyapatite. Ceramics

International 29-6, 2003, 699-706.

[20] G. Muralithran, S. Ramesh. The effects of sintering temperature on the properties of hydroxyapatite, 2000. Ceram. Int. 26, 221230. 